\title{
Corela
}

Cognition, représentation, langage

2-2 | 2004

Vol. $2, n^{\circ} 2$

\section{Polysémie lexicale et représentation géométrique du sens : l'exemple du verbe entendre}

\section{Corinne Ozouf}

\section{OpenEdition}

\section{Journals}

Édition électronique

URL : http://journals.openedition.org/corela/622

DOI : $10.4000 /$ corela.622

ISSN : 1638-573X

\section{Éditeur}

Cercle linguistique du Centre et de I'Ouest - CerLICO

Référence électronique

Corinne Ozouf, « Polysémie lexicale et représentation géométrique du sens : l'exemple du verbe entendre », Corela [En ligne], 2-2 | 2004, mis en ligne le 15 décembre 2004, consulté le 02 mai 2019. URL : http://journals.openedition.org/corela/622 ; DOI : 10.4000/corela.622

Ce document a été généré automatiquement le 2 mai 2019.

\section{(c) (i) (2)(2)}

Corela - cognition, représentation, langage est mis à disposition selon les termes de la licence Creative Commons Attribution - Pas d'Utilisation Commerciale - Partage dans les Mêmes Conditions 4.0 International. 


\title{
Polysémie lexicale et représentation géométrique du sens : l'exemple du verbe entendre
}

\author{
Corinne Ozouf
}

\section{Présentation}

1 Le Dictionnaire Electronique des Synonymes (D.E.S.), élaboré au CRISCO et en libre consultation sur internet (www.crisco.unicaen.fr), permet de visualiser dans un espace sémantique multidimensionnel l'ensemble du potentiel sémantique d'une unité lexicale par le calcul de la distance sémantique entre tous ses synonymes.

2 L'outil est lié aux données lexicographiques : ce dictionnaire est obtenu après compilation et symétrisation des renvois synonymiques contenus dans sept dictionnaires ${ }^{1}$. La structure du dictionnaire est formalisée en un graphe dont les sommets sont les entrées du dictionnaire et dont les arêtes sont les relations synonymiques. Le logiciel VISUSYN utilise ce graphe pour construire automatiquement un espace géométrique multidimensionnel ${ }^{2}$.

3 A travers l'exemple du verbe entendre, cet article offre une illustration du mode d'exploitation du D.E.S. où plusieurs niveaux d'analyse se dégagent et veut montrer en quoi il constitue un outil d'exploration privilégié de la polysémie lexicale. Nous verrons que la matérialisation du sens fait apparaître le continuum de signification dans ses nuances les plus fines et tout particulièrement les points d'articulation favorisant son déploiement, et que la représentation géométrique obtenue automatiquement est interprétable, les synonymes se répartissant en fonction de critères sémantiques et syntaxiques. 


\section{Exploitation de la page de résultats de la requête entendre}

4 La page de résultats offre une première approche du sens. Elle fournit un ensemble d'informations qui sont interprétables du point de vue de l'organisation du sémantisme du mot-vedette et qui permettent d'anticiper sur ce à quoi va pouvoir ressembler la répartition du sens sur la visualisation.

5 Le D.E.S. propose une liste des synonymes associés à entendre et les répartit en composantes connexes c'est-à-dire en groupes tels que chaque unité d'un groupe est synonyme d'au moins une autre unité de ce groupe. Les synonymes d'une même composante sont un signe de polysémie où s'inscrit un continuum de signification et plusieurs composantes sont un signe d'homonymie: il n'y a aucun élément de sens commun.

6 S'ensuit la liste des cliques ( $c$ f. annexe 01 (fin de la page)) qui constituent des sousensembles d'unités toutes synonymes les unes des autres donnant le détail des nuances de sens prises par le mot-vedette et permettant par là même d'apprécier le continuum de signification à l'œuvre dans les composantes connexes ${ }^{3}$.

entendre : 41 synonymes et 54 cliques

\subsection{Liste des synonymes}

7 admettre, apprécier, appréhender, assister, attraper, auditionner, avoir une idée, bien prendre, comprendre, compter, concevoir, confesser, connaître, consentir, cultiver, désirer, discerner, distinguer, écouter, embrasser, exaucer, exiger, goûter, interpréter, juger, ouïr, pénétrer, percevoir, posséder, pratiquer, préférer, prétendre, réaliser, reconnaître, saisir, satisfaire, sentir, se rendre compte, souhaiter, voir, vouloir.

\subsection{Liste des composantes connexes}

1. admettre, apprécier, appréhender, assister, attraper, comprendre, compter, concevoir, confesser, connaître, consentir, cultiver, discerner, distinguer, embrasser, exaucer, goûter, interpréter, juger, ouïr, percevoir, posséder, pratiquer, préférer, pénétrer, reconnaître, réaliser, saisir, satisfaire, se rendre compte, sentir, voir, écouter

2. auditionner (cf. clique 22)

3. avoir une idée ( $c f$. clique 23 ) non prises en compte dans la visualisation

4. bien prendre (cf. clique 24)

5. désirer, exiger, prétendre, souhaiter, vouloir (cf. clique 47)

8 On observe pour entendre cinq composantes dont trois ne sont constituées que d'un seul synonyme (composantes 2, 3,4). Celles-ci correspondent à des sens isolés qui dans les dictionnaires n'ont pas dû être mis en relation avec d'autres synonymes que entendre luimême. Ils sont d'emblée écartés et ne figurent d'ailleurs pas sur la visualisation.

9 La première composante comporte la grande majorité des synonymes et l'examen des synonymes contenus dans cette composante révèle à la fois des effets de sens concrets liés à l'audition et d'autres abstraits de l'ordre de la compréhension. Il existe donc un 
continuum de signification entre un saisissement par l'oreille et un saisissement intellectuel.

10 La composante 5, constituée des synonymes 'désirer, exiger, prétendre, souhaiter, vouloir' est assez particulière: si elle présente effectivement une suite de synonymes, ceux-ci ne représentent pas un continuum de signification puisqu'ils sont synonymes entre eux, ils se recouvrent totalement et apparaissent conjointement dans une seule clique (clique 47).

11 Si cette composante renvoie à un effet de sens isolé, elle ne peut néanmoins subir le traitement réservé aux trois composantes supprimées, dans la mesure où il s'agit d'un emploi typique $\mathrm{du}$ verbe, relativement fréquent, notamment dans le registre journalistique (une étude menée sur le corpus du Monde catégorisé par le logiciel TREE TAGGER montre que $30 \%$ des 3925 occurrences de entendre relèvent de cette valeur).

Cette composante 5 correspond à la valeur typique d'entendre liée à la volition, valeur qui se construit en co-texte avec deux structures syntaxiques qui lui sont exclusives :

- un infinitif avec co-référence du sujet : le sujet exprime sa détermination

(1) De nombreux cadres entendaient réduire la police qui commençait à écraser le PC. (Le Monde 1995)

- une complétive au subjonctif : l'acte de volonté s'exerce sur autrui.

(2) - ah! Je vous préviens, une fois pour toutes, que je défends au jardinier de vendre ou de donner à quiconque des légumes. Je sais bien qu'il faut en faire trop pour en avoir assez... et que les trois quarts se perdent... tant pis !... j'entends qu'on les laisse se perdre... (Mirbeau, Le Journal d'une femme de chambre, p.321)

On peut s'interroger sur les raisons d'une répartition en partie homonymique des valeurs de entendre, sachant que d'une manière générale les articles lexicographiques et les travaux sémantiques préconisent un traitement polysémique pour ce verbe.

Un premier élément de réponse est que les emplois du verbe en construction pronominale ne figurent pas dans les résultats, ils sont traités à part. Or, J.Picoche (1986) considère que certains emplois pronominaux sont une transition entre intellection et volition, en particulier s'entendre pour signifiant «se mettre d'accord pour réaliser qqc, mettre au point un projet ». Cet ajustement sur le sens à donner est intermédiaire entre «saisir ce qui est donné à comprendre » et « déterminer un dessein à l'exclusion d'un autre ».

Par ailleurs, et c'est plus surprenant, il manque aux résultats le synonyme vouloir dire pourtant cité dans le Larousse et le Robert ${ }^{4}$, emploi courant et diversement traité selon les sources. Ainsi, J.Picoche, en terme de continuité, le situe là encore de manière intermédiaire et, dans son dictionnaire de langue ${ }^{5}$, le regroupe avec les emplois volitifs. Le TLFi le place à la fin du domaine intellectuel et J.J.Franckel et D.Lebaud (1990) l'incluent résolument dans le fonctionnement volitif, vouloir dire marquant un centrage qualitatif, une sélection parmi des possibles qui élimine l'altérité.

Cette diversité de traitement et ce lien manquant (vouloir dire) manifestent l'instabilité, la fragilité du lien entre la valeur intellectuelle et la valeur volitive par rapport à celui qui unit les valeurs sensorielle et intellectuelle : la stabilisation interprétative de l'entendu se fait dans le cadre de la perception (concrète ou abstraite) pour les valeurs sensorielle et intellectuelle tandis qu'elle se fait dans le cadre de la conception (construction d'un objet de pensée) pour la valeur volitive. L'homonymie serait une «propriété émergente » de fragmentation du champ sémasiologique en réseaux de sens autonomes selon la terminologie de B.Victorri. 
l'espace sémantique » présent dans la page de résultats. Le logiciel VISUSYN utilise l'ensemble des cliques pour construire automatiquement l'espace géométrique multidimensionnel : dans cet espace, le sens du mot-vedette est représenté par un nuage de points, chaque point correspondant à une gouttelette de sens ou clique. Les synonymes sont représentés par un contour qui englobe les cliques où l'on trouve ce synonyme. l'autre de vaste déploiement pour la composante 1. synonyme à des effets de sens ou cliques. Le synonyme en tête de classement couvre ainsi une partie importante de l'espace sémantique, représente un sens large puisqu'il apparaît dans le plus grand nombre de cliques. Dans le cas de entendre, on constate avec étonnement que le synonyme en tête de classement n'est autre que voir, un autre verbe de perception ( $c f$. Tableau 1).

\section{Cf. annexe 02 (fin de la page)}

Voir étant un verbe d'une perception autre qu'auditive, cela sous-entend que pour un rapprochement sémantique avec entendre, il prend majoritairement un sens abstrait. C'est donc un effet de sens abstrait qui représente le plus largement entendre (20 cliques : 1, 5, $6,8,10,20,25,26,27,30,33,34,37,39,41,44,45,51,53,54)$.

Cette prépondérance d'effets de sens associés à la valeur intellectuelle trouve sans doute son explication dans l'originalité du parcours historique d'entendre, dont la structure sémantique s'est trouvée profondément bouleversée. En ancien français, entendre était un verbe essentiellement intellectuel et abstrait. Il a hérité de la valeur auditive du monosème oür (extrêmement marginalisé aujourd'hui) tandis que comprendre, à l'origine rare et relatif à la contenance, s'est emparé d'une partie des effets de sens intellectuels d' entendre ${ }^{6}$.

21 Notons que les premiers synonymes du classement (voir, connaitre, comprendre, percevoir, pénétrer) sont relativement proches sémantiquement. En réalité, ils se chevauchent partiellement ce qui n'a rien d'étonnant si l'on considère qu'il y a pour entendre plus de cliques (54 nuances de sens) que de synonymes (41). La consultation de la liste des cliques confirme ce chevauchement puisque dans nombre de cas les premiers synonymes s'associent (ex: clique 27 \{comprendre, connaître, embrasser, entendre, pénétrer, voir\}). A l'inverse, les synonymes en fin de classement dénotent des sens précis.

Ce classement va donc du sens large vers le sens étroit en terme de recouvrement synonymique d'entendre. Du point de vue de la visualisation, le classement est éclairant puisque le sens large d'entendre, capable de rassembler de multiples variations sémantiques, aura plutôt tendance à se situer vers le centre (le point d'intersection des axes), au moins dans le plan 1 腥 2 , tandis que les sens spécifiques vont s'en écarter, jusqu'à figurer aux extrémités des axes ${ }^{7}$.

\section{Exploitation des plans de visualisation du D.E.S.}

Le deuxième niveau d'exploitation du dictionnaire s'active par le lien «Visualisation de sont représentés par un contour qui englobe les cliques où l'on trouve ce synonyme. 
La visualisation géométrique dispose les différents effets de sens du verbe selon leur voisinage sémantique de manière à représenter le mieux possible la distance sémantique entre eux.

25 L'espace sémantique est multidimensionnel et la visualisation proposée retient les deux premières dimensions ${ }^{8}: c^{\prime}$ est le plan offrant la meilleure représentation de l'ensemble de la distribution des cliques, où les distinctions principales sont repérables. Il est de ce fait incontournable. Mais il est possible de faire «tourner » la représentation, notamment lorsque ce premier plan ne suffit pas à faire apparaittre toutes les acceptions ou du moins leur déploiement.

La méthode adoptée consiste donc à favoriser le plan primaire, même en cas d'espace fortement disjoint, pour dégager l'ensemble des composantes, à se servir du zoom pour se faire une idée des cliques extrêmes du noyau central, puis à changer de plan afin de suivre le destin de ces cliques et éventuellement d'en faire apparaître de nouvelles, devenues extrêmes par la rotation.

\subsection{Le plan primaire de entendre}

Dans le plan $1 \times 2$ ( $c$ f. Figure 1), la répartition d'ensemble des effets de sens de entendre montre distinctement 3 zones autour des axes. La lecture de cette répartition se fait dans le sens des aiguilles d'une montre en partant en haut à droite.

8 En zone 1 (en haut à droite), l'extrémité est visible et coïncide avec la clique \{entendre, exaucer, satisfaire, écouter\} qui désigne une zone spécifique aux emplois sensoriels.

29 La signification se déploie le long de l'axe et on note, outre une progression du sens précis vers le sens large au centre, une progression du sens le plus agentif ${ }^{9}$ vers le moins agentif: exaucer / satisfaire $\rightarrow$ écouter $\rightarrow$ ouir $\rightarrow$ percevoir.

Cf. annexe 03 (fin de la page)

Sur toute cette zone, le sujet est animé et le complément concret. Notons également que le co-texte est davantage contraint à l'extrémité qu'au centre. Ainsi, exaucer implique un sujet désignant une autorité divine et un complément nominal désignant un humain ou traduisant une demande

(3) - c'est vous, Caroline, qui, déjà, prétendez en faire un petit clerc..., comme vous faites de votre Dieudonné un olibrius de savant... - sans doute, et que Dieu m' entende! (Adam, L'Enfant d'Austerlitz, p.121)

tandis que percevoir admet un sujet animé (humain ou animal) et un complément concret (tout ce qui peut être audible) aux multiples variantes formelles :

- SN

(4) J'entendais les grives dans le lierre du peuplier carolin (Mauriac)

- proposition infinitive

(5) On entendit Médor aboyer à la grille, furieux, derrière le bruit d'une voiture côtoyant le saut de loup. (Adam, L'Enfant d'Austerlitz, p.76)

- $\mathrm{N}+$ relative

(6) ...On n'entend que le vent qui souffle là rude et violent,...(Pesquidoux)

- complétive

(7) Ensuite j'entendis que monsieur s'essuyait, se frottait, s'ébrouait... (Mirbeau, Le Journal d'une femme de chambre, p.108) 
3 illisible en tant que telle et où il est difficile de percevoir une extrémité. Cependant, la
présence du synonyme confesser, auquel correspond la clique \{admettre, confesser, entendre, reconnaître\} laisse penser que se situent là les emplois non sensoriels du verbe.

Insistons sur le rôle éclairant de la clique, qui, si elle précise le sens spécifique du mot vedette, précise également le sens des synonymes qui lui sont associés: la sélection sémantique est mutuelle. De fait, confesser n'a pas de rapport avec un emploi auditif du type "entendre qqn en confession" mais prend un sens abstrait comme admettre le suggère. entendre (proximité avec comprendre, saisir, connaittre...). Plus précisément, voir, dans un sens abstrait est l'expression du saisissement de quelque chose porteur de signification. Les emplois relevant de cet effet de sens sont du type " entendre ce que qqn veut dire " ou «j'entends (bien)». Dans un sens concret, voir est évidemment limité et désigne une attitude de réceptivité face à un contenu significatif. Il est d'ailleurs associé à écouter dans la clique. C'est donc le resserrement autour d'une parole chargée de sens qui sert de liaison.

En zone 3 (en bas à gauche), figure la composante 5 , très éloignée du centre et sans lien avec le reste. Cette disposition est peu satisfaisante car elle limite le déploiement de sens de la zone 1, ceci étant dû à la distance de la zone 3 par rapport aux autres.

Avant de changer de plan, il est important de signaler que sur aucun autre nous n'aurons de vue pleinement satisfaisante des deux composantes simultanément :

- soit la cinquième se trouve superposée à la première alors qu'en réalité elles n'ont aucun lien,

- soit les composantes sont distinctes mais les emplois sensoriels et intellectuels de la composante 1 sont superposés.

Afin d'avoir un premier aperçu de la zone 2, il est possible d'effectuer un zoom sur cette zone (Figure 2), ce qui met en évidence trois orientations de sens autour de voir, celles-ci ayant en commun dans leur déploiement respectif de passer d'une compréhension passive à une compréhension active jusqu'à impliquer le sujet dans ses actes.

\section{Cf. annexe 04 (fin de la page)}

En partant des extrémités, les trois axes sont :

- cultiver $\rightarrow$ pratiquer

- préfére $\rightarrow$ distinguer $\rightarrow$ voir

- consentir $\rightarrow$ admettre

- confesser 
épartition d'ensemble est schématisée sur la figure 1. Compte-tenu de cette répartition, il est logique d'avoir un espace vide en haut à gauche de la visualisation : il n'existe pas de zone de conception concrète.

\subsection{Le changement de plan}

41 La fonction " choix des axes " permet de sélectionner le plan offrant une visualisation satisfaisante du déploiement de sens de la composante 1 . Ainsi, les axes $4 \times 2$ redistribuent dans l'espace à la fois les emplois sensoriels et les emplois intellectuels (mis en évidence par la pratique du zoom). Les multiples effets de sens s'y organisent de la manière suivante (cf. Figure 3) :

Comme la schématisation superposée à la visualisation l'indique, la partie supérieure concerne les emplois sensoriels du verbe qui chevauchent l'axe vertical - zone 1 / extrémité $1=$ \{entendre, exaucer, satisfaire, écouter $\}$ tandis que la partie inférieure est réservée aux emplois intellectuels qui se déploient largement à gauche et à droite selon des orientations sémantiques distinctes.

point de vue du co-texte, le passage de la partie supérieure à la partie inférieure se traduit par des restrictions: le sujet devient strictement humain et le complément, désormais abstrait, est formellement plus contraint (l'entendu n'étant plus de nature événementielle mais conceptuelle, certaines constructions sont exclues, comme la proposition infinitive ou la relative, à l'inverse emploi de l'interrogative indirecte). Et plus on s'éloigne de la partie centrale pour aller vers les extrémités, vers les effets de sens spécifiques, plus les options co-textuelles se réduisent c'est-à-dire que la correspondance sens / structure se fait plus étroite.

Au centre figure voir, commun à l'ensemble des orientations sémantiques et synonyme de transition concret/abstrait.

Le déploiement de la zone intellectuelle étant plus lisible, si l'on souhaite préciser le sens pour entrevoir les diverses orientations, on recourt à percevoir, le seul autre synonyme commun aux emplois concrets et abstraits et qui couvre moins largement la zone intellectuelle.

En zone 2 (en bas à droite), percevoir établit un lien direct avec l'orientation autour de distinguer. Cette zone évoque le simple saisissement du sens et son déploiement correspond à un ajustement interprétatif autour de ce qui est donné à comprendre avec, à l'extrémité (extrémité $2=$ \{distinguer, entendre, préférer $\}$ ), un jugement interprétatif, une appréciation. Il existe donc une progression impliquant une prise de position.

Cf. annexe 05 (fin de la page)

Le continuum de signification est assuré sur la zone 3 (partie inférieure gauche) par le synonyme concevoir. La particularité de cette zone 3 est de comporter deux orientations sémantiques :

48

- l'une, directement en contact avec concevoir, évoque le positionnement intellectuel avec un principe d'adhésion (admettre), qui peut d'ailleurs n'être que préalable comme l'indique l'extrémité \{admettre, confesser, entendre, reconnaître\} qui correspond à la structure entendre bien que...(mais) et annonce un argument contraire. 
(8) J'entends bien que l'esclavage et le servage ont été éliminés de la propriété individuelle. Mais les radicaux peuvent-ils avoir l'assurance que tout élément de servitude, d'oppression, d'injustice, en a disparu ? (Jaurès, Etudes socialistes, p.153) synonymique d'admettre: \{admettre, confesser, entendre, reconnaître\} (extrémité 3) d'une part et \{admettre, consentir, entendre\} (extrémité 4) d'autre part, emploi vieilli de la construction transitive indirecte.

50 - l'autre, où réaliser relaie concevoir, est liée à la pratique (pratiquer) avec, à l'extrémité (extrémité $5=$ \{cultiver, entendre, pratiquer\}), une accentuation de l'intérêt porté à cette pratique: cultiver, c'est entretenir ou approfondir la pratique. Cette orientation correspond en particulier au rapport sens / construction : entendre qqc à , ne rien entendre à / « comprendre parfaitement une méthode et savoir l'appliquer ».

51 Contrairement à ce que pourrait laisser penser la visualisation, les synonymes réaliser et admettre ne se recouvrent pas. Le recours aux cliques permet d'éviter ce genre d'erreur d'interprétation: dans aucune d'elles ne figurent conjointement les deux synonymes. En réalité, la composante 1 prend la forme d'un tétrapode.

Ainsi, l'élément de sens commun aux deux orientations sémantiques de la zone 3 est l'adhésion intellectuelle tandis que l'élément distinctif est l'opposition entre l'adhésion sur le sens et l'adhésion dans la pratique.

La lecture des plans de visualisation confirme les informations délivrées dans la page de résultats sur la structuration du sens et présente tout particulièrement l'avantage de représenter le continuum de signification. Certaines cliques servent de transition entre des valeurs typiques parfois très éloignées les unes des autres et mettent en lumière l'ensemble des paramètres favorisant la progression sémantique sur les axes.

54 Les plans de visualisation soulignent de manière automatisée le caractère central de la valeur intellectuelle dans l'organisation du sens du verbe entendre. Le verbe voir, activé du côté intellectuel et couvrant largement l'espace sémantique, sert non seulement d'interface entre les valeurs sensorielle et intellectuelle mais se trouve également à la base d'une diversification riche (la majorité des synonymes de entendre relèvent de la valeur intellectuelle) et complexe du sens intellectuel.

\section{L'exploitation de la matrice de co-appartenance des synonymes aux cliques du verbe entendre}

55 La matrice de co-appartenance des synonymes aux cliques, outil d'exploitation avancée, est accessible dans les pages internes du site du CRISCO. Elle doit être saisie dans un fichier texte, qui est ouvrable sur tableur EXCEL.

Cette matrice (cf. annexe 06 (fin de la page)) fournit le calcul du degré de cooccurrence des synonymes entre eux autrement dit leur degré de proximité sémantique. Elle permet donc une analyse détaillée des liens synonymiques qu'entretiennent mutuellement les synonymes et se révèle fort utile en cas de difficulté de lecture de la visualisation.

57

La visualisation, rappelons-le, se présente sous la forme d'un nuage de points dont l'enjeu est de faire apparaitre les éléments de sens articulant l'organisation sémantique du mot vedette.

Corela, 2-2 | 2004 
La difficulté de lecture se concentre aux alentours de la zone centrale, étant donné que les extrémités sont parfaitement lisibles. Cette difficulté rencontrée notamment avec le verbe entendre prend l'aspect d'une zone de «tuilage » de cliques c'est-à-dire une zone de fort regroupement de signification où les sens se recouvrent largement.

Il est important de pouvoir analyser de telles zones dans la mesure où elles sont le signe d'un changement progressif de signification. Pour identifier la zone (il peut y en avoir plusieurs) de concentration de signification, il suffit de repérer le synonyme possédant le degré de co-appartenance le plus élevé qui figure dans la colonne «total » de la matrice. Pour entendre, c'est voir qui, avec un total de 63 cooccurrences, localise la zone de « tuilage ».

Une fois ce repérage effectué, on procède à la lecture horizontale (ou verticale) de la matrice, lecture capitale qui nous apprend de quoi est constituée la zone de « tuilage » de cliques. Les synonymes les plus cooccurrents avec voir figurent en rouge foncé sur la ligne du synonyme voir et il est intéressant de constater que notre intuition première ( $C f$. \$2) se trouve confirmée à savoir que les synonymes les plus cooccurrents avec voir sont ceux qui figurent juste après lui dans le classement des synonymes c'est-à-dire connaitre (cooccurrence 8), comprendre (7), percevoir (7), saisir (7) et concevoir (7).

61 Donc, du point de vue de la visualisation, la matrice nous apprend que les premiers synonymes du classement se superposent largement. Un des avantages de cette microanalyse est la levée d'ambiguïté. En effet, si voir est le synonyme qui couvre le plus largement les emplois de entendre, il est alors le plus polysémique et ne permet pas de cerner précisément les différences de sens de entendre : la lecture de la matrice y remédie puisqu'elle redistribue les effets de sens à l'intérieur de voir ( $C f$. les synonymes de cooccurrence élevée cités).

2 On notera que dans le total, n'est pas pris en compte le chiffrage en diagonale relatif à la fréquence, dans la mesure où il correspond au degré de cooccurrence d'un synonyme avec lui-même. Cette information n'est pas pour autant négligeable puisqu'elle chiffre le classement des synonymes fourni dans la page de résultats sous forme de graphique.

Si l'on compare le classement des synonymes à la matrice de co-appartenance des synonymes aux cliques, il apparait que le classement reflète le degré d'extension des synonymes par rapport à entendre, (le synonyme en tête de classement est celui qui s'étend le plus largement dans l'espace sémantique) tandis que la matrice de coappartenance concerne à l'opposé le degré de chevauchement des synonymes entre eux, elle reflète la concentration de signification (la plus forte cooccurrence localise le recouvrement de sens).

4 La matrice identifie donc la zone de concentration de signification, de quoi est constituée cette zone et également comment en sortir c'est-à-dire là où s'amorce le déploiement de signification.

Il faut pour ce faire procéder du large à l'étroit : au lieu de s'intéresser aux synonymes de cooccurrence élevée avec voir, on se concentre au contraire sur ceux de faible cooccurrence, sachant qu'ils vont se situer à l'extrémité de la zone synonymique de voir (peu de recouvrement). Les synonymes de faible cooccurrence figurent en rouge clair sur la ligne du synonyme voir. Si l'on prend une cooccurrence de 1 avec voir, nous sélectionnons les synonymes admettre, assister et écouter. La lecture de la matrice en diagonale (chiffrage du classement) permet de vérifier si ces synonymes sont bien des transitions sachant que chacun des synonymes doit avoir une fréquence supérieure à 1 . 

une représentation du sens dont l'intérêt tant pédagogique que scientifique est de disposer simultanément et selon le rapport qu'ils entretiennent - c'est-à-dire leur proximité de sens - l'ensemble des synonymes représentatifs du potentiel sémantique du mot-vedette.

71 Il offre en outre l'avantage d'être utilisable sur plusieurs niveaux, selon le degré d'affinement sémantique souhaité. L'utilisateur va ainsi privilégier le dégroupement des synonymes en séparations homonymiques ou pousser plus loin l'analyse sémantique en recherchant une dissociation plus fine des sens.

72 Comme l'analyse du verbe entendre l'a montré, les visualisations géométriques, ainsi que le recours à la matrice qui en facilite et en précise la lecture, sont d'un grand intérêt pour la recherche en sémantique dans la mesure où y figurent à la fois discontinuité de sens c'est-à-dire la possibilité de repérer des orientations de sens et continuité sémantique puisque l'on observe le changement graduel de signification le long des axes. La meilleure illustration de ce continuum est fournie par la clique qui, assurant la jonction entre deux zones synonymiques, fait partie des deux zones à la fois sans être représentée par l'une à l'exclusion de l'autre. Cette micro- zone est propre aux cumuls de valeurs et emplois intermédiaires tels qu'ils sont décrits par B.Victorri et C.Fuchs. recours aux composantes connexes, lesquelles traduisent un dégroupement homonymique, fournira des éléments de réponse à la question de la séparation ou non de l'item en homonymes. A l'inverse, l'exploration des zones de tuilage de cliques permettra d'opérer des regroupements de signification. Autre avantage, la fusion des dictionnaires à l'origine du D.E.S. ayant fait disparaitre tout commentaire, les visualisations constituent « un terrain neutre dans la controverse entre sémanticiens sur la notion de dérivation de sens $»^{10}$. 


\section{BIBLIOGRAPHIE}

Blumenthal, P. (2003), « Histoire cognitive d'un verbe 'mental' : comprendre » in J.E. Tyvaert (dir. 2003), La cognition dans le temps. Tübingen, Niemeyer, pp. 27-40.

Franckel, J.J. \& Lebaud, D. (1990), Les Figures du sujet. Paris, Ophrys.

François, J. \& Manguin, J.L. (2004), « La polysémie adjectivale entre synonymie et sélection contextuelle : le cas de propre ». In H. BOUILLON (éd.), Langues à niveaux multiples. Hommage au Professeur Jacques Perrot à l'occasion de son éméritat (BCILL 112). Louvain : Peeters.

François, J., Manguin, J.L., Eufe, R., Fesenmeier, L., Ozouf, C. \& Sénéchal, M. (2004), Le Dictionnaire Electronique des Synonymes : un mode d'emploi à trois niveaux. Cahier du CRISCO 17, téléchargeable sur le site www.crisco.unicaen.fr.

Kahlmann, A. (1975), Traitement automatique d'un dictionnaire de synonymes. Stockholm, Université de Stockholm.

Manguin, J.L. (à paraître en 2004), « Regroupements de synonymes par indices de similitude : exemple avec l'adjectif ancien ». Communication au colloque Les adjectifs non prédicatifs, 28-29 novembre 2002, Université de Paris-Nord Villetaneuse. A paraître dans les Cahiers de Lexicologie.

Manguin, J.L. (à paraître), Utilisation d'un corpus catégorisé pour l'étude et la représentation de la synonymie en contexte. Actes des $3^{\text {èmes }}$ journées de linguistique de corpus, 11-13 septembre 2003, Université de Bretagne Sud, Lorient.

Manguin, J.L. \& Victorri, B. (1999), Représentation géométrique d'un paradigme lexical, actes de la conférence TALN 1999, pp. 363-368.

Picoche, J. (1986), Structures sémantiques du lexique français. Paris, Nathan.

Picoche, J. « Ouïr, entendre, comprendre, une vue psychomécanique sur le renouvellement du lexique » in Etude de lexicologie et dialectologie, rassemblées par Nelly Andrieux-reix et Geneviève Hasenohr, Conseil International de la Langue Française, 1995, article n²6 pp. 265-276.

Picoche, J. \& Rolland, J.C. (2001), Le Dictionnaire du Français Usuel. Bruxelles, Duculot.

Ploux, S. (1997), Modélisation et traitement informatique de la synonymie. Linguisticae Investigationes, XXI, Amsterdam, John Benjamins.

Victorri, B. \& Fuchs, C. (1996), La polysémie : une construction dynamique du sens, Paris, Hermès.

\section{NOTES}

1. Les relations de synonymie proviennent de deux dictionnaires analogiques (le Grand Larousse et le Grand Robert), deux dictionnaires des synonymes du $19^{\mathrm{e}}$ (Lafaye et Guizot) et trois dictionnaires des synonymes du $20^{\mathrm{e}}$ (Bailly, Bénac et Du Chazaud). Le fait de fusionner sept dictionnaires rend l'outil beaucoup plus consensuel comme l'a fait remarquer Kahlmann dans sa thèse (1975). Le choix de la méthodologie a été discutée dans Manguin (2004) où l'on s'aperçoit que les regroupements de synonymes obtenus par les simples données syntagmatiques (occurrences dans un corpus) sont moins pertinents que ceux obtenus avec les données 
paradigmatiques ( issues d'un dictionnaire de synonymes) ou encore mieux, de la moyenne des deux.

2. La méthode de construction de l'espace sémantique a été décrite et discutée dans Ploux (1997).

3. Pour le détail de l'exploitation du graphe, se reporter à la section $2 \mathrm{du}$ Cahier du Crisco $\mathrm{n}^{\circ} 17$ (juillet 2004) téléchargeable sur le site www.crisco.unicaen.fr.

4. Le Grand Larousse de la langue française en 7 volumes, édition 1971 et le Grand Robert de la langue française, dictionnaire alphabétique et analogique en 6 volumes, édition 1983.

5. Picoche, J. \& Rolland, J.J. (2001), Le Dictionnaire du Français Usuel, Bruxelles, Duculot.

6. Cf. J.Picoche (1995) et P.Blumenthal (2003).

7. Il s'avère en fait que pour entendre, le synonyme voir se situe systématiquement au centre, quel que soit le plan sélectionné.

8. Les axes sont le résultat d'une Analyse en Composantes Principales.

9. Sens où s'exerce la détermination du sujet.

10. François, J. p.29 du Cahier du Crisco ${ }^{\circ} 17$.

\section{RÉSUMÉS}

Le Dictionnaire Electronique des Synonymes offre une représentation automatisée du champ sémasiologique des unités lexicales qui se fonde sur une approche dynamique et continuiste de la construction du sens. Cet article présente l'ensemble des outils mis à disposition dans ce dictionnaire pour l'exploration du sens, outils qui offrent un intérêt tant pédagogique que scientifique, leur observation permettant une analyse fine et objective de la polysémie lexicale.

The Electronic Dictionary of Synonyms presents an automated representation of the polysemy of lexical units which is based on a dynamic and continuous model of meaning construction. This paper deals with the tools implemented by this dictionary. The interest of these tools is both pedagogical and scientific as their observation presents a fine and objective analysis of lexical polysemy.

\section{INDEX}

Mots-clés : polysémie, dictionnaire, synonymes, représentation du sens, traitement automatique, sémantique

\section{AUTEUR}

\section{CORINNE OZOUF}

Université de Caen - CRISCO (FRE 2805) 